\title{
Durch attraktive Karrierewege Public Health in Deutschland nachhaltig stärken - Kernforderungen an eine Public-Health- Strategie für Deutschland im Bereich Human Resources
}

\section{Sustainable Strengthening of Public Health in Germany through Attractive Career Paths - Core Requirements for a Public Health Strategy for Germany in the Area of Human Resources}

\author{
Autoren \\ Franziska Hommes ${ }^{1}$, Katharina Alpers ${ }^{2}$, Birgit Reime ${ }^{3}$, Ute Rexroth ${ }^{2}$
}

Institute

1 Nachwuchsnetzwerk Öffentliche Gesundheit, Berlin

2 Abteilung für Infektionsepidemiologie, Robert Koch Institut, Berlin

3 Abteilung für angewandte Gesundheitswissenschaften, Hochschule Furtwangen, Furtwangen

\section{Schlüsselwörter}

Public Health, Human Resources, Nachwuchsfachkräfte, Karrierewege, Zukuftsforum Public Health

Key words

Public Health, young professionals, career paths, Future forum Public Health, human resources

Bibliografie

DOI https://doi.org/10.1055/a-1082-0890

Online-Publikation: 7.2.2020

Gesundheitswesen 2020; 82: 303-305

(c) Georg Thieme Verlag KG Stuttgart · New York

ISSN 0941-3790

Korrespondenzadresse

Dr. Ute Rexroth

Fachgebiet 32 (Surveillance)

Robert Koch-Institut

Seestrasse 10

13353 Berlin

rexrothu@rki.de

\section{ZUSAMMENFASSUNG}

Der Bedarf an qualitativ gut ausgebildeten Public-HealthFachkräften nimmt angesichts einer gesteigerten Bedeutung von Gesundheitsförderung und Prävention in Deutschland zu. Demgegenüber gestalten sich viele Karrierewege im Bereich Public Health zu intransparent, was für interessierte Nachwuchskräfte zusätzliche Hürden schafft und zur bestehenden Lücke zwischen Bedarf und Angebot beiträgt. Vor dem Hintergrund internationaler Rahmenwerke sowie der konkreten Arbeitsmarktsituation in Deutschland diskutierte die AG 11 „Karrierewege in Public Health“ des 3. Symposiums des Zukunftsforums Public Health aktuelle Herausforderungen und formulierte erste Lösungsansätze.

\begin{abstract}
The demand for a well-trained public health workforce is increasing in view of the increased importance of health promotion and disease prevention in Germany. However, many career paths in public health are non-transparent, which creates additional barriers for interested young professionals and contributes to the existing gap between demand and supply. Against the backdrop of international frameworks and the specific labor market situation in Germany, the working group 11 "Career Paths in Public Health" of the $3^{\text {rd }}$ Symposium of the Future Forum Public Health discussed current challenges and formulated initial solutions.
\end{abstract}

Interdisziplinarität ist Grundlage von Public Health

Der Einsatz der vielfältigen Möglichkeiten, die Gesundheit der Bevölkerung zu schützen und zu fördern, erfordert die Mitwirkung unterschiedlichster Disziplinen und Professionen. Das im Bereich Public Health tätige Personal setzt sich dementsprechend interdis- ziplinär und interprofessionell zusammen und schließt je nach individuellem Betätigungsfeld u. a. Fachkräfte aus den Bereichen Gesundheitswissenschaften, Human-, Veterinär- und Zahnmedizin, Psychologie, Pharmazie, Pflege, Rettungsdienst, Geburtshilfe, medizinische Fachberufe, Biologie, Epidemiologie, Soziologie, Kommunikation, Statistik, aber auch angrenzenden Gebiete wie Geografie, Jura oder Stadtplanung mit ein. Public Health adressiert in 
erster Line den Gesundheitsschutz und die Gesundheitsförderung auf Bevölkerungsebene, baut aber auf dem allgemeinen Gesundheitswesen auf und setzt deswegen auch dort ausreichend qualifiziertes Personal voraus. Ein gemeinsames Verständnis der Methoden und Inhalte von Public Health kann im Rahmen diverser Aus-, Fort- und Weiterbildungen sowie grundständiger und postgradualer Studiengänge erworben werden.

\section{Diskussion um eine Public-Health-Strategie zur Förderung von Human Resources}

Die vielfältigen Ausbildungsangebote entwickeln sich dezentral, spezialisieren sich in unterschiedliche Schwerpunkte und orientieren sich teilweise ausschließlich direkt am lokalen Arbeitsmarkt. Eine zentrale Bedarfsplanung und strategische Steuerung in Bezug auf Public-Health-Fachkräfte findet bislang in Deutschland nicht statt. Internationale Public-Health-Rahmenwerke von $\mathrm{WHO}^{1}$ und $\mathrm{ECDC}^{2}$ fordern allerdings eine übergreifende nationale multisektorale Strategie für die Förderung und Ausbildung von Fachkräften im Bereich Public Health (public health workforce). Die aktuell gelebte Praxis in Deutschland weicht bislang von dieser Forderung ab.

Die völkerrechtlich bindenden und von der 58. Weltgesundheitsversammlung verabschiedeten Internationalen Gesundheitsvorschriften (IGV) (2005) zielen darauf ab, potenziell grenzüberschreitende Gefahren biologischen, chemischen oder radionuklearen Ursprungs, zu verhüten, frühzeitig zu erkennen und diesen zu begegnen. Sie wurden geschaffen, um gesundheitliche Notlagen mit potenziell internationaler Tragweite bereits am Ursprungsort zu erkennen und einzudämmen. Um die Umsetzung der IGV zu gewährleisten, ist ein starkes öffentliches Gesundheitswesen erforderlich und effektive Personalressourcen werden explizit als eine von 13 Kernkapazitäten (WHO 2018) definiert. In der jährlichen Evaluation in Form einer Selbsteinschätzung Deutschlands wird der Bereich personelle Ressourcen u. a. aufgrund der fehlenden Strategie am schlechtesten bewertet ${ }^{3}$.

Die internationale Forderung nach einer übergreifenden Strategie wird durch die praktische Erfahrung im eigenen System unterstützt: Das Dickicht der Qualifikationsangebote und intransparente Karrierewege erschweren die Orientierung für Nachwuchskräfte und Absolventinnen und Absolventen der Ausbildungs- und Studiengänge, aber auch für Lehrende und potenzielle Arbeitgeber. Eine übergreifende strategische Planung und die Verständigung auf Kerncurricula der jeweiligen Qualifikationswege erscheinen vor diesem Hintergrund sinnvoll und dringend erforderlich.

1 WHO: Joint external evaluation tool: International Health Regulations (2005), second edition. Geneva: WHO (2018). Im Internet: https://www. who.int/ihr/procedures/joint-external-evaluations/en/; Stand: 04.08.2019; WHO: State Party self-assessment annual reporting tool. Geneva: WHO (2018). Im Internet: https://extranet.who.int/sph/ihr-monitoring-evaluation/spar/1219; Stand: 04.08.2019.

2 HEPSA - health emergency preparedness self-assessment tool, user guide'. Stockholm: ECDC (20189 Im Internet: https://ecdc.europa.eu/en/ publications-data/hepsa-health-emergency-preparedness-self-assessment-tool; Stand 04.08.2019.

3 WHO: Strategic Partnership for International Health Regulations (2005) and Health Security (SPH). Im Internet: https://extranet.who.int/sph/ihrmonitoring-evaluation; Stand: 04.08.2019.

\section{Aktuelle Herausforderungen}

Eine zentrale Herausforderung einer solchen Strategieentwicklung für Public-Health-Fachkräfte stellt die belastbare Datengrundlage zur aktuellen Bedarfsermittlung dar. Eine gute, zukunftsweisende Strategie fußt auf einer aktuellen Bestands- und Bedarfsanalyse. Es fehlen aber konkrete und aktuelle Angaben dazu, wie die personelle Ausstattung im Bereich Public Health aufgestellt ist. Wie viele Menschen arbeiten in Deutschland in diesem Fachbereich? Welche Altersstruktur liegt vor? Wie sind sie qualifiziert? Wer sind die Arbeitgeber? Welchen Qualifikationsbedarf gibt es in unterschiedlichen Bereichen und Professionen? Wo drohen in Zukunft Lücken oder wo bestehen diese bereits heute? Darauf, dass im gesamten Gesundheitswesen bis 2030 mit einer Verschärfung des Fachkräftemangels gerechnet werden muss, weist auch ein Gutachten zum Fachkräftebedarf im Gesundheits- und Sozialwesen 2030 hin, das 2018 vom RWI-Leibniz-Institut für Wirtschaftsforschung im Auftrag des Sachverständigenrates zur Begutachtung der gesamtwirtschaftlichen Entwicklung erstellt wurde ${ }^{4}$. Schon 2015 stellte ein gemeinsamer Bericht der Kultus- und Gesundheitsministerkonferenz fest: „Angesichts der zunehmenden und teils dramatischen personellen Engpässe bei gleichzeitig ständig wachsenden Aufgaben und Herausforderungen für den Öffentlichen Gesundheitsdienst (ÖGD) sind dringend Maßnahmen erforderlich, um seine Funktionsfähigkeit zu sichern" 5 . Auch die 91. Gesundheitsministerkonferenz 2018 begrüßte in Anwesenheit des Bundesgesundheitsministers das „Leitbild für einen modernen Öffentlichen Gesundheitsdienst“6. Darin heißt es u. a. „Die Verbindung des Öffentlichen Gesundheitsdienstes mit der Wissenschaft muss sowohl in der Forschung als auch in der Aus- und Weiterbildung sowie in der Praxis gestärkt werden“. Seither hat sich die Lage nicht verbessert. Aus dem ÖGD liegen zahlreiche Einzelberichte zu Engpässen vor v. a. aus Gesundheitsämtern wird von einem Mangel an Nachwuchskräften berichtet ${ }^{7}$ - welcher aber nicht mit aktuellen und bundesweit repräsentativen Zahlen quantifiziert werden kann.

\section{Internationale Rahmenwerke und nationale Akteure als Anknüpfungspunkte}

International anerkannte Instrumente wie das Joint External Evaluation Tool (JEE) zur Überprüfung der Umsetzung der IGV oder das Essential Public Health Operations (EPHOS)-Selbsteinschätzungs-

4 RWI - Leibniz-Institut für Wirtschaftsforschung: Fachkräftebedarf im Gesundheits- und Sozialwesen 2030 (2018). Im Internet: www.sachverstaendigenrat-wirtschaft.de/fileadmin/dateiablage/gutachten/jg201819/ arbeitspapiere/Arbeitspapier_06-2018.pdf; Stand: 04.08.2019.

5 Gemeinsamer Bericht der Gesundheitsministerkonferenz und der Kultusministerkonferenz „Fachkräftesicherung im Gesundheitswesen“ 2015. Im Internet: https://www.kmk.org/fileadmin/Dateien/veroeffentlichungen_beschluesse/2015/2015_06_12-Fachkraeftesicherung-im-Gesundheitswesen.pdf; Stand: 11.08.2019.

6 Länderoffene Projektgruppe „Leitbild ÖGD“, 20.02.2018. Im Internet: https://www.lgl.bayern.de/gesundheit/sozialmedizin/oegd_handbuch/ doc/leitbild_oegd.pdf; Stand: 11.08.2019.

7 RBB 24, Ärztemangel im Gesundheitsdienst Finanzsenator besteht auf außertariflicher Lösung, 10.12. 2018. Im Internet: https://www.rbb24. de/politik/beitrag/2018/12/berlin-protest-aerzte-oeffentlicher-gesundheitsdienst.html; Stand: 16.08.2019 
instrument des Europäischen Regionalbüros der $\mathrm{WHO}^{8}$ könnten als Unterstützung bei der Bestandsanalyse dienen. Diese internationalen Rahmenwerke sind zwar nicht speziell auf das föderale System Deutschlands ausgerichtet, sie können aber einen wichtigen Orientierungs- und Handlungsrahmen bieten, der an die spezifische Situation in Deutschland adaptiert werden sollte. Dabei muss auch die differenzierte Akteurslandschaft Deutschlands berücksichtigt werden. Einrichtungen mit Relevanz für Lehre, Aus-, Fortund Weiterbildung sind u. a. Universitäten, Fachhochschulen, Schools of Public Health, Akademien der Bundesländer, oberste Bundes- und Landesbehörden für Gesundheit, Bundesinstitute wie das Robert-Koch-Institut (RKI), das Bundesinstitut für Risikobewertung (BfR), das Umweltbundesamt (UBA) oder das Bundesamt für Bevölkerungsschutz und Katastrophenhilfe (BBK), Ärztekammern, Kassenärztliche Vereinigungen, Krankenkassen, Betriebe, usw. In Anbetracht der Vielzahl der für die Entwicklung der gemeinsamen Zukunftsstrategie relevanten Institutionen bleibt die Frage offen, welche Institutionen die Federführung des Prozesses übernehmen könnten oder sollten.

\section{Karrierewege im Öffentlichen Gesundheitsdienst}

An Ideen für die Inhalte fehlt es indessen nicht. Primäres Ziel sollte die langfristige Förderung effektiver und hochqualitativer PublicHealth-Fachkräfte auf lokaler, regionaler und nationaler Ebene sein. Ein Ausbau der personellen und finanziellen Kapazitäten im ÖGD ist dafür eine entscheidende Voraussetzung. Die Attraktivität des ÖGD sollte durch eine attraktivere Aus- und Weiterbildung, wettbewerbsfähige Gehälter ${ }^{9}$ und transparente Karrierewege gesteigert werden. Dabei sollten bspw. Famulaturen im Medizinstudium sowie die Weiterbildung zum Facharzt für Öffentliches Gesund- heitswesen ${ }^{10}$ stärker beworben und gefördert werden. Zudem sollte die Öffnung des ÖGD auf Ebene des Gesundheitsamtes für die Breite der gesundheitswissenschaftlichen Berufsgruppen gefördert werden. Modelle aus anderen Ländern können hierbei als Vorbilder dienen, wie etwa die Ausbildung zum Public-Health-Consultant in Großbritannien. Um Qualität und Vergleichbarkeit zu verbessern, sollten in der Strategie Schlüsselqualifikationen, etwa in Form von Kerncurricula, für Public-Health-AbsolventInnen auf Bachelor- und Masterebene definiert werden. Eine Kooperation von Gesundheitswissenschaftlerlnnen/Public Health mit angrenzenden Professionen (z. B. Medizin, Sozialwissenschaften, Stadtplanung) sollte ausgebaut werden.

Zusammenfassend fehlt in Deutschland bislang eine gemeinsame Strategie zur Ausbildung und Förderung von Public-HealthFachkräften, obwohl verfügbare und gut ausgebildete Arbeitskräfte eine Voraussetzung für die Erreichung anderer Public-Health-Ziele sind und ihre Förderung somit essentieller Bestandteil einer umfassenden Public Health-Strategie sein muss. Die Entwicklung einer solchen Strategie, die mit messbaren und zeitgebundenen Indikatoren verknüpft sein sollte, könnte zu gesteigerter Transparenz, einheitlicher Qualifikationsstandards und verbesserter Koordination in der Ausbildung von Public-Health-Fachkräften führen. Die in der Strategie enthaltenen Maßnahmen sollten sich an europäischen und internationalen Strategien messen und neben der Bundesebene auch die Bundesländer und Kommunen umfassen.

Interessenkonflikt

Die Autorinnen/Autoren geben an, dass kein Interessenkonflikt besteht.

8 WHO: Self-assessment tool for the evaluation of essential public health operations in the WHO European Region (2015). Im Internet: http:// www.euro.who.int/en/publications/abstracts/self-assessment-tool-forthe-evaluation-of-essential-public-health-operations-in-the-who-european-region-2015; Stand: 04.08.2019.

9 BZÖG: 89. Gesundheitsministerkonferenz: Für einen starken Öffentlichen Gesundheitsdienst (2016). Im Internet: https://www.bzoeg.de/aktuellesleser/items/89-GMK-2016.html; Stand: 04.08.2019.

10 Bundesärztekammer, (Muster-)Weiterbildungsordnung 2018. Im Internet: https://www.bundesaerztekammer.de/fileadmin/user_upload/downloads/ pdf-Ordner/Weiterbildung/MWBO-16112018.pdf; Stand: 04.08.2019. 